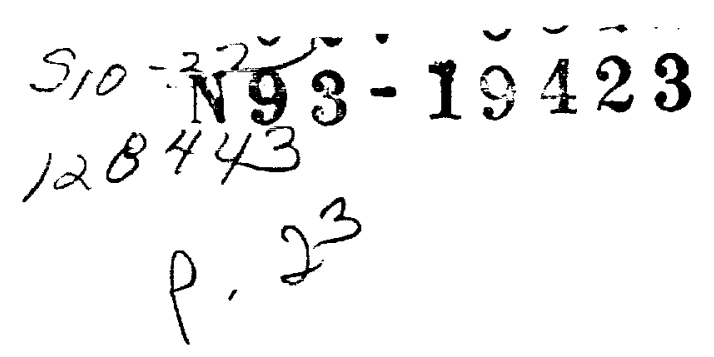

\title{
A Method for Modeling Discontinuities in a Microwave Coaxial Transmission Line
}

\author{
T. Y. Otoshi \\ Ground Antennas and Facilities Engineering Section
}

\begin{abstract}
This article presents a method for modeling discontinuities in a coaxial transmission line. The methodology involves the use of a nonlinear least-squares fit program to optimize the fit between theoretical data (from the model) and experimental data. When this method was applied to modeling discontinuities in a slightly damaged Galileo spacecraft $S$-band $(2.295-G H z)$ antenna cable, excellent agreement between theory and experiment was obtained over a frequency range of $1.70-2.85 \mathrm{GHz}$. The same technique can be applied for diagnostics and locating unknown discontinuities in other types of microwave transmission lines, such as rectangular, circular, and beam waveguides.
\end{abstract}

\section{Introduction}

The Galileo spacecraft, launched on October 18, 1989, is currently on its interplanetary journey to encounter Jupiter in 1995. One of the important experiments to be performed with this spacecraft in 1993 is the gravitational wave experiment to support Einstein's General Theory of Relativity.

Prior to launch, Galileo underwent environmental testing in the Space Simulator at JPL. After these tests, it was discovered that the S-band output power had dropped about $0.2 \mathrm{~dB}$ at the transmit frequency of $2.295 \mathrm{GHz}$. More alarming were the radical changes observed in the subsequent measured insertion loss versus frequency characteristics of the S-band antenna cable. Instead of the small peak-to-peak sinusoidal variations seen previously on the pre-environmental test frequency response curves, numerous nonperiodic humps and valleys (of unusual amplitudes) were seen on the post-environmental test curves.
This author (consulting on an emergency basis) diagnosed the problem and provided a satisfactory mathematical model of the slightly damaged cable. This model depicted the Galileo cable as having developed crimps at two different cable clamp locations, and reasonable agreement between theory and experiment was obtained. Further analysis indicated that if the magnitudes of discontinuities doubled during Galileo's interplanetary journey to Jupiter, an additional $0.2-\mathrm{dB}$ loss increase would occur at the $2.295-\mathrm{GHz}$ transmit frequency, but this worst-case expected loss increase (0.4-dB total degradation) would still be acceptable.

Based upon the author's satisfactory explanation of the phenomenon, the Galileo Project decided that the S-band cable did not have to be replaced, and the spacecraft was shipped to Cape Canaveral on schedule. The decision not to replace the cable was partially based upon the fact that $\mathrm{X}$-band $(8.415 \mathrm{GHz})$ was the prime data channel for ra- 
dio science experiments and S-band was less important. Now that S-band has become the prime data channel due to problems with the X-band high-gain antenna, the expected S-band antenna performance characteristics should be reanalyzed.

The purpose of this article is to transfer the technology gained from the successful modeling of the discontinuities in the Galileo S-band antenna cable. This technique can be applied to obtaining models of discontinuities in other types of transmission lines, including rectangular, circular, and beam waveguides. Once a good analytical model is developed, it can then be used for diagnostic purposes or for analytical studies of worst-case situations. For example, models of transmission lines with discontinuities can be used to determine the effects of multiple reflections that are known to degrade the frequency stability and noise temperature performance of a receiving system.

The writing of this article was also motivated by the desire to provide radio scientists and gravitational wave experimenters with an accurate model of the damaged $S$ band antenna cable on Galileo. Such a model might assist in the error analyses of radio science data, should the need arise in the near future. Discontinuities in a cable give rise to multiple reflections that affect Doppler phase, frequency, and group delay stability. Cable movement and cable temperature changes can cause the phases of individual reflected waves to change, thereby affecting the overall phase and amplitude of the output signal. Continued flexing of the cable during Galileo's interplanetary journey to Jupiter can also make the magnitudes of the individual reflections worse. An accurate model can help to establish error bounds (on radio science data) associated with worst-case signal level and frequency stability degradation situations.

In the following sections, the methodology is described and then demonstrated by applying it to the Galileo cable problem for which experimental data were already available. Comparisons are given showing results obtained with a trial-and-error method versus the proposed new method.

\section{Previous Models of the Galileo Cable}

As was described in a 1989 report, ${ }^{1}$ Model 1 was the first trial model that was derived. It consisted of shunt susceptances (representing discontinuities) separated by different lengths of coaxial line. The first necessary step

\footnotetext{
1 T. Y. Otoshi, "Galileo Cable Study Report," JPL Interoffice Memorandum 3328-89-0108, (internal document), Jet Propulsion Laboratory, Pasadena, California, May 17, 1989.
}

of the modeling procedure was to obtain time domain plots from measured $S$-parameters over a frequency range centered at the particular frequency of interest. The $S$ parameters for the Galileo cable were obtained with the HP $8510 \mathrm{~B}$ automatic network analyzer. Then the shunt susceptance magnitudes ${ }^{2}$ and approximate locations of discontinuities in the cable were estimated from the return loss-time domain plots.

When the unaltered values (extracted directly from return loss-time domain plots) were used in Model 1, the agreement between theoretical and experimental data was poor. The parameters for the subsequently developed Model 2 were almost the same as those of Model 1, except that the line lengths between discontinuities were adjusted slightly to cause all the individual reflection coefficients to add up in phase at the input port at $2310 \mathrm{MHz}$. As can be seen in Fig. 1, Model 2 provided satisfactory agreement between theory and experimental data. Figure 2 shows the equivalent circuit for Model 2. From this equivalent circuit, it was determined that a total of four discontinuities existed near the connector regions and cable clamp locations (see Fig. 3).

The agreement between theory and experiment for Model 2 was considered to be satisfactory (even very good) in 1989. However, it was known then that the model was incomplete because at least one more discontinuity had to be added near one of the connectors. This was known because when all discontinuities were removed, except those representing the connectors, the periodicity of the insertion loss versus frequency curve was wrong, and therefore the agreement between the calculated and the preenvironmental cable test data was not good. It appeared that adding one nore discontinuity in the connector region closer to the end of the cable would have resulted in a better model. However, further attempts to develop improved models after adding an additional discontinuity and then readjusting the line lengths (by trial and error) for a good fit proved to be excessively time-consuming and fruitless. It was clear that some type of least-squares fitting program was required to obtain a better model. The effort to obtain a better model was temporarily abandoned.

\section{Computer Program}

Although not tasked to find an improved model for the Galileo cable, the author continued working on the problem on a low-priority basis because of his desire to learn how to perform curve fits between mathematical models

\footnotetext{
${ }^{2}$ See Eqs. (A-3) and (A-4) in Appendix A.
} 
and experimental data. It is common practice just to fit experimental data with polynomial curves because a polynomial curve-fitting process is easy to perform and useful for displaying trends and calculating intermediate values. What is not generally known, as has been pointed out by C. Lawson of JPL, is that it is almost as easy to perform curve fits between experimental data and the theoretical results from any mathematical model. If the physical phenomenon associated with the experimental data can be modeled mathematically (no matter how involved and complex), then curve fitting can be done between theoretical and experimental data through the use of a nonlinear least-squares fit (NLSF) program. Variance, correlation coefficients, and standard deviations can also be easily computed from the residuals of the nonlinear curve-fitting process.

A linear model is defined here as one whose coefficients $a_{i}$ can be expressed explicitly in the form

$$
\begin{aligned}
y(x)= & a_{0}+a_{1} f_{1}(x)+a_{2} f_{2}(x)+\cdots \\
& +a_{i} f_{i}(x)+\cdots+a_{n} f_{n}(x)
\end{aligned}
$$

Polynomials are a subset of the general linear form given by the above Eq. (1). A nonlinear model differs in that the coefficients to be solved for (best-fitted) can be expressed within any of the expressions for $f_{i}(x)$ or in almost any mathematical form. As long as the program steps can be written to calculate values of $y(x)$ for input values of $x$, then a nonlinear least-squares fit can be performed to solve for the unknown coefficients.

With Lawson's assistance, a subroutine computer program was written to find a best-fit model of the Galileo cable by using the existing International Mathematical Scientific Library (IMSL) NLSF computer program already available on the UNIVAC at JPL in 1989. The problem with that program was that it did not allow the user to specify bounds on the parameters to be adjusted and bestfitted. Occasionally the IMSL program gave best-fit parameter results that were not physically realizable.

After further consultation with Lawson, it became clear that JPL needed an improved NLSF program that could be used for various types of ongoing modeling problems at JPL. To fulfill this immediate need, Lawson performed an extensive search of available NLSF programs in industry and academic institutions. As a result, he recommended the use of a particular NLSF program ${ }^{3}$ that was later de-

\footnotetext{
${ }^{3}$ This program was a later version of the original release of NL2SOL by David Gay and Linda Kaufman, now at AT\&T Bell Laboratories.
}

scribed in another JPL publication [1]. This program was unlike most available least-squares fit programs in that it allowed the user to specify bounds on the parameters to be best-fitted. This program has the additional advantages of being public domain software and can be run on a personal computer. The input data required for this program are (1) the measured data; (2) the theoretical values for the mathematical model calculated from a subroutine; and (3) estimates of the nominal, upper, and lower bound values of the parameters to be best-fitted.

The Fortran subroutine ultimately developed for the Galileo cable-modeling study calculated the overall Sparameters for a cable that had seven or more discontinuities separated from each other by lengths of coaxial line whose attenuation constant and relative dielectric constant could be specified. Program steps were written to calculate the $S$-parameters of a basic network consisting of a shunt discontinuity and a length of lossy line. The equations for two types of shunt discontinuities used in this study are given in Appendix A. Another subroutine was written to compute the overall $S$-parameters of two 2-port networks that were cascaded. When the overall $S$-parameters of two basic networks are cascaded, the cascaded network becomes the equivalent 2-port that is cascaded with the next basic network. The overall $S$-parameters are again calculated and stored. This procedure was repeated until all seven basic networks were cascaded.

The insertion loss in decibels of the final overall network was then calculated from

$$
I L=20 \log _{10}\left|S_{21}\right|
$$

where $S_{21}$ is the $S$-parameter for the transmission coeffcient of the overall cascaded network when terminated in a nonreflecting load [2]. The insertion loss is computed at each frequency to form the theoretical data set for the model. Then comparisons are made with the experimental insertion loss data file which was read into the program. The parameters to be adjusted were (1) the discontinuity magnitudes (in terms of shunt susceptance or capacitance values) and (2) the line lengths separating the discontinuities. The program finds the parameter values (within the specified bounds) that give the best fit (using a leastsquares convergence criterion) between theoretical and experimental values. Even though the distances between the discontinuities were allowed to be adjusted within specified bounds, the program was written so that the resulting overall length of the cable for the model had to be equal to the actual physical length of the cable. 


\section{Improved Models}

After the development of Model 2, it was known that additional discontinuities in the cables existed near the connector interfaces. Examinations of detailed drawings of the connector regions revealed locations of potential discontinuities that were not taken into account in Model 2. Figure 4 shows potential discontinuities as being the back ends (or sharp edges) of the rigid Kynar sleeves located about 2.9 inches from the faces of the cable connectors. Bending of the cable at these points can cause crimps or make permanent deep creases on the outer diameter. Another type of potential discontinuity occurs at the connecting regions (Fig. 5) where there are changes in diameter dimensions and dielectric materials within the coaxial transmission line.

When the NLSF program (described in Section III) became available, new models were developed that included the above-described discontinuities. Two likely equivalent circuits for the cable discontinuities are (1) shunt capacitances whose susceptance values are functions of frequencies and (2) capacitive shunt susceptances whose values do not change over the frequency of interest. The models corresponding to these two types of discontinuities are denoted as Models 3 and 4 and are described below.

\section{A. Model 3}

Model 3 represents the cable discontinuities as constant shunt capacitances. The constant shunt capacitance might occur in practice when the reduced outer diameter of the cable is squashed over some physical length (e.g., the cable clamp width). For this type of discontinuity, the magnitude of $S_{11}$ of the individual discontinuity changes with frequency. For this model with nominal values, the final best-fit parameters determined by the NLSF program are shown in Fig. 6. Note that the locations of discontinuities of the model occur very close to actual locations of the cable clamps, the edges of the Kynar sleeve, and the connector discontinuities.

Figure 7 shows the comparison between theoretical and measured insertion losses. It can be seen that the agreement is significantly better than that of the Model 2 fitted curve shown in Fig. 1. To obtain such a good fit, it was necessary to use accurate values of cable attenuation due to line losses between the shunt discontinuities. Appendix B describes the procedure that was used to accurately determine cable attenuation (minus connectors). Although Model 3 appears to be an excellent model, it was found to be incorrect. As may be seen in Fig. 8, when all the discontinuities are removed except the two outer connector discontinuities, the agreement between theory and the pre-environmental test data becomes progressively worse at the higher frequencies.

\section{B. Model 4}

Model 4 is based upon representing the discontinuities as constant susceptance values over the frequency range of interest. This type of discontinuity could be a deep crease, or crimp, on the outer diameter of the cable. Such a discontinuity in practice can be created by bending the cable against the edge of a cable clamp or the edge of a $\mathrm{Ky}$ nar sleeve. This type of discontinuity can be represented as two shunt capacitances separated by a short distance (less than 0.005 wavelength, or a single shunt susceptance, as discussed in Appendix C). The overall equivalent circuit and locations of the discontinuities along the cable are shown in Fig. 9. Model 4 is the result of best-fitting 15 parameters (seven discontinuities and eight line lengths) by using 101 frequency points for a frequency range of 1.7 to $2.85 \mathrm{GHz}$. The Model 4 discontinuity locations, shown in Fig. 9, correspond very closely with the actual locations of cable clamps, the edges of the Kynar sleeve, and internal discontinuities of the connectors. The locations of the modeled discontinuities are estimated to be within \pm 0.5 in. of the actual cable discontinuities. As with Model 3, the line losses between discontinuities for Model 4 were properly accounted for.

It can be seen in Fig. 10 that the theoretical values for this model agree well (within $\pm 0.02 \mathrm{~dB}$ ) with experimental data. Good agreement between theoretical and experimental return losses was also obtained, as can be seen in Figs. 11-12. Figure 13 shows that when all internal discontinuities except the two outer discontinuities are removed, the agreement between the model and pre-environmental test data is still excellent.

To ensure that Model 4 is, in all respects, the correct model of the cable, the phases of $S_{21}$ were considered, as well as the magnitudes. However, attempts to fit both magnitudes and phases to experimental data were unsuccessful. Also, the attempt to do similar least-squares fitting to the $S_{11}$ and $S_{22}$ experimental data proved unsuccessful. It was later revealed that special adapters had been used to measure the reflection coefficients $S_{11}$ and $S_{22}$. When the effects of the adapters were gated out, ${ }^{4}$ the measurement planes might not have been properly referred back to the connector interface planes of the cable. ${ }^{5}$

\footnotetext{
${ }^{4}$ See Hewlett Packard 8510B Operating Manual for a discussion of gating techniques for the purposes of removing external discontinuity effects from the measured $S$-parameter data.

${ }^{3}$ W. Folwell, personal communication, Spacecraft Telecommunications Equipment Section, Jet Propulsion Laboratory, Pasadena, California, 1990.
} 
It is difficult to best-fit phase data if the measurement and model reference planes do not coincide.

Due to the difficulty of obtaining a good fit between the theory and the model, based on both $S$-parameter amplitude and phase data, another method was used to verify the model. This method compared experimental and theoretical time domain plots. Time domain plots require both magnitude and phase information over a wide frequency range. Figures 14-16 show time domain plots for $S_{21}, S_{11}$, and $S_{22}$ data, respectively. Good agreement was obtained for $S_{21}$, but for the $S_{11}$ and $S_{22}$ time domain plots, the reference planes had to be shifted by an amount approximately equal to the lengths of the special adapters used in the measurement. The necessity to shift reference planes for $S_{11}$ and $S_{22}$ is consistent with the possibility that the measurement plane might not have been correctly referenced back to the cable connector interface planes.

Despite the described difficulties with the reference plane problem associated with the $S_{11}$ and $S_{22}$ time domain plots, good agreement was obtained for the $S_{21}$ time domain plot. One can conclude from the data presented that Model 4 is an excellent representation of the Galileo $S$-band cable after the environmental tests.

The sharp edges of the cable clamps and the Kynar sleeves are the probable causes of the discontinuities on the outer diameter of the cable. As was stated in the previously cited report ${ }^{6}$ the cable clamps should be redesigned

${ }^{B}$ T. Y. Otoshi, op. cit. so that crimping or creasing will not occur when the cable is bent against the clamps. It was also stated that only a 0.02 -in. reduction in outer diameter of the Galileo cable could produce discontinuities of the magnitudes presented in the models studied. For future spacecraft cables, the edges of the currently rigid Kynar sleeves should also be redesigned and be made flexible.

\section{Conclusions}

A model has been found that gives excellent agreement between theory and experiment for the Galileo spacecraft $\mathrm{S}$-band antenna cable. The current model now has seven discontinuities (including connectors) instead of four obtained for the previous model.

The excellent results described would not have been possible without the use of the NLSF program. This program was easy to use, and formal documentation ${ }^{7}$ is now available. Engineers are encouraged to use this program for their modeling work.

The method presented here was demonstrated for a coaxial cable, but the technique can be extended to the modeling of discontinuities in other types of transmission lines, such as rectangular, circular, and beam waveguides (with a shroud).

7 Math77, Release 4.0, A Library of Mathematical Subprograms for Fortran 77, JPL D-1341, Rev. C (internal document), Jet Propulsion Laboratory, Pasadena, Califomia. May 1992.

\section{Acknowledgments}

Dr. C. Lawson, at that time supervisor of the Applied Mathematics Group at JPL, graciously supplied the public domain NLSF software and provided assistance with the coding procedures. Scott Stewart, a contractor from PRC in Pasadena, California, programmed the HP 8510B Network Analyzer computer to read theoretical $S$-parameter data into data files for the purpose of generating theoretical time domain plots. It should be pointed out that this capability does not exist using software available from Ilewlett Packard. Technical discussions with Kent Kellogg and Phil Stanton of JPL were helpful. 


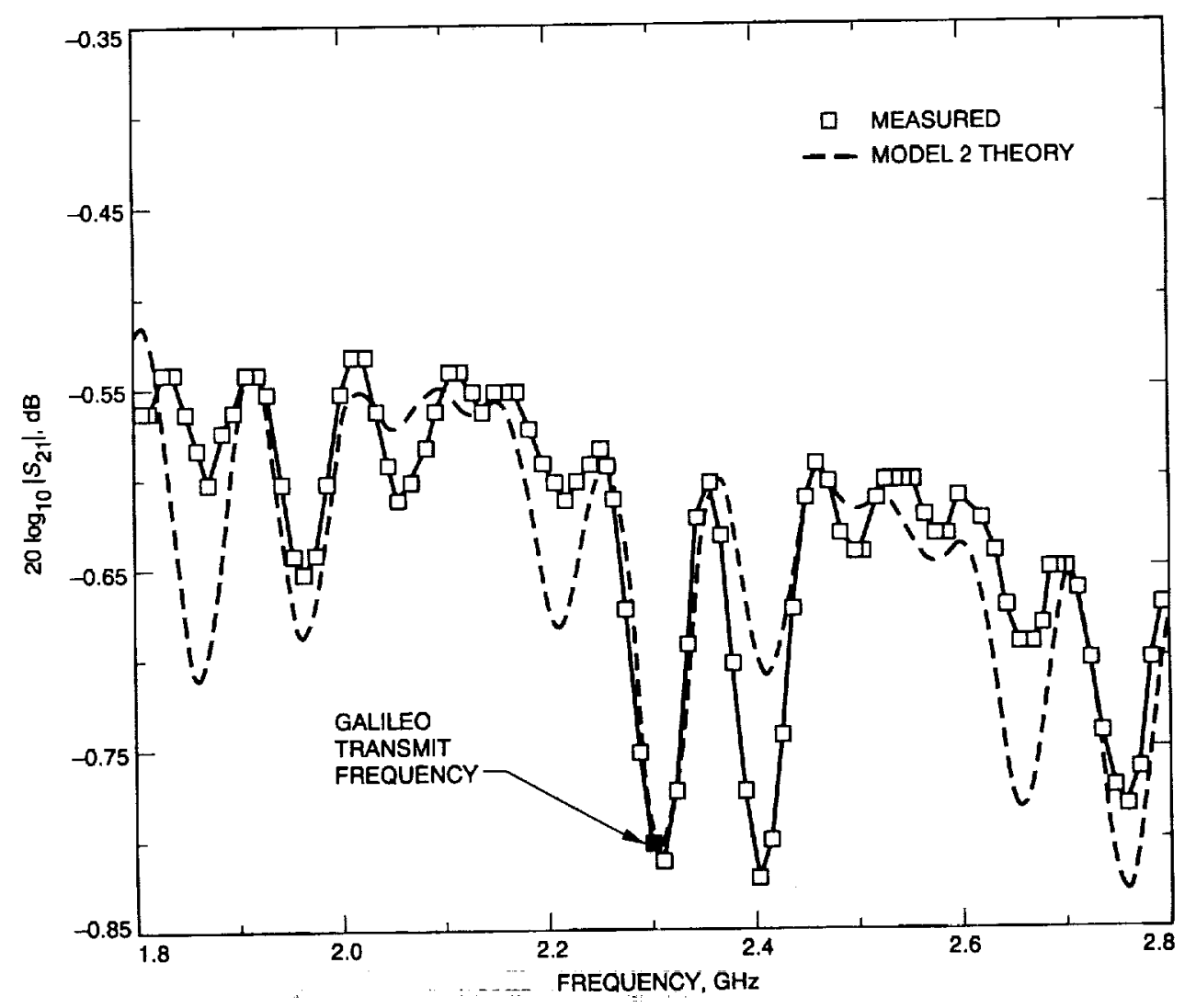

Flg. 1. Comparison of experimental and Model 2 theoretical data. 


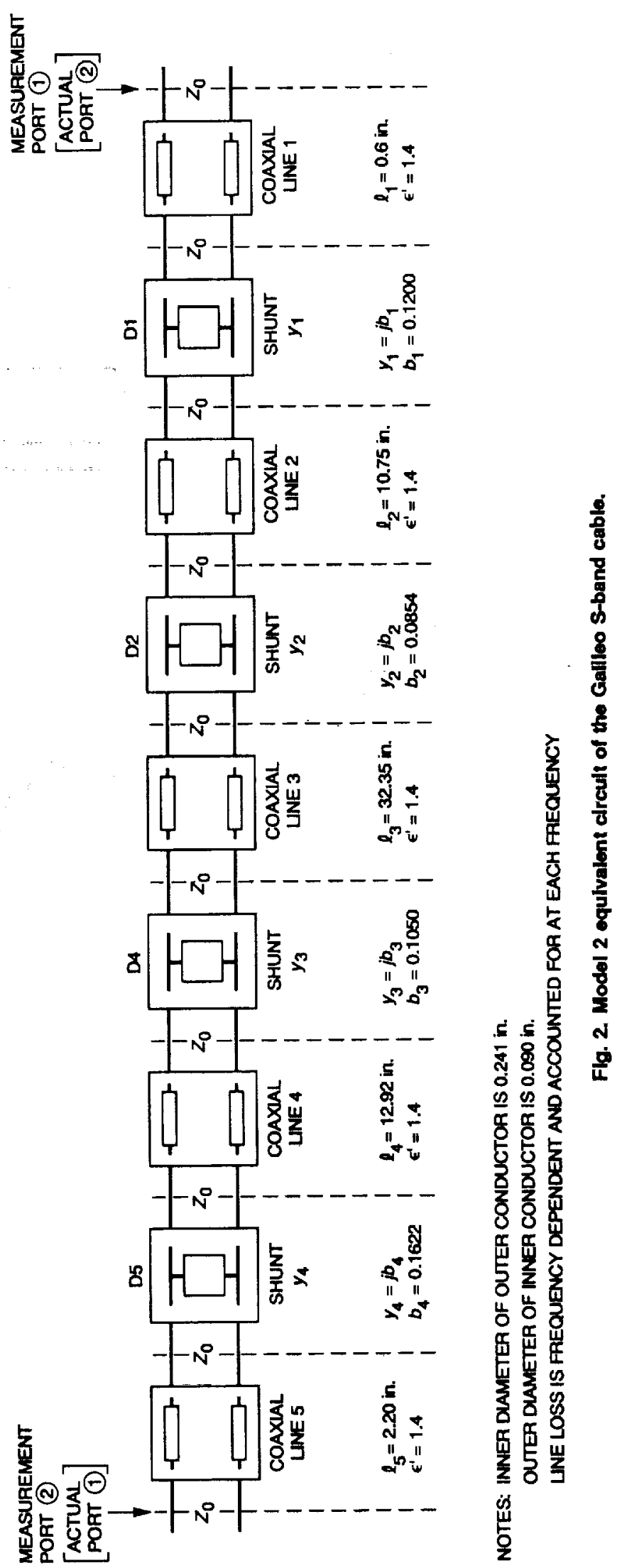




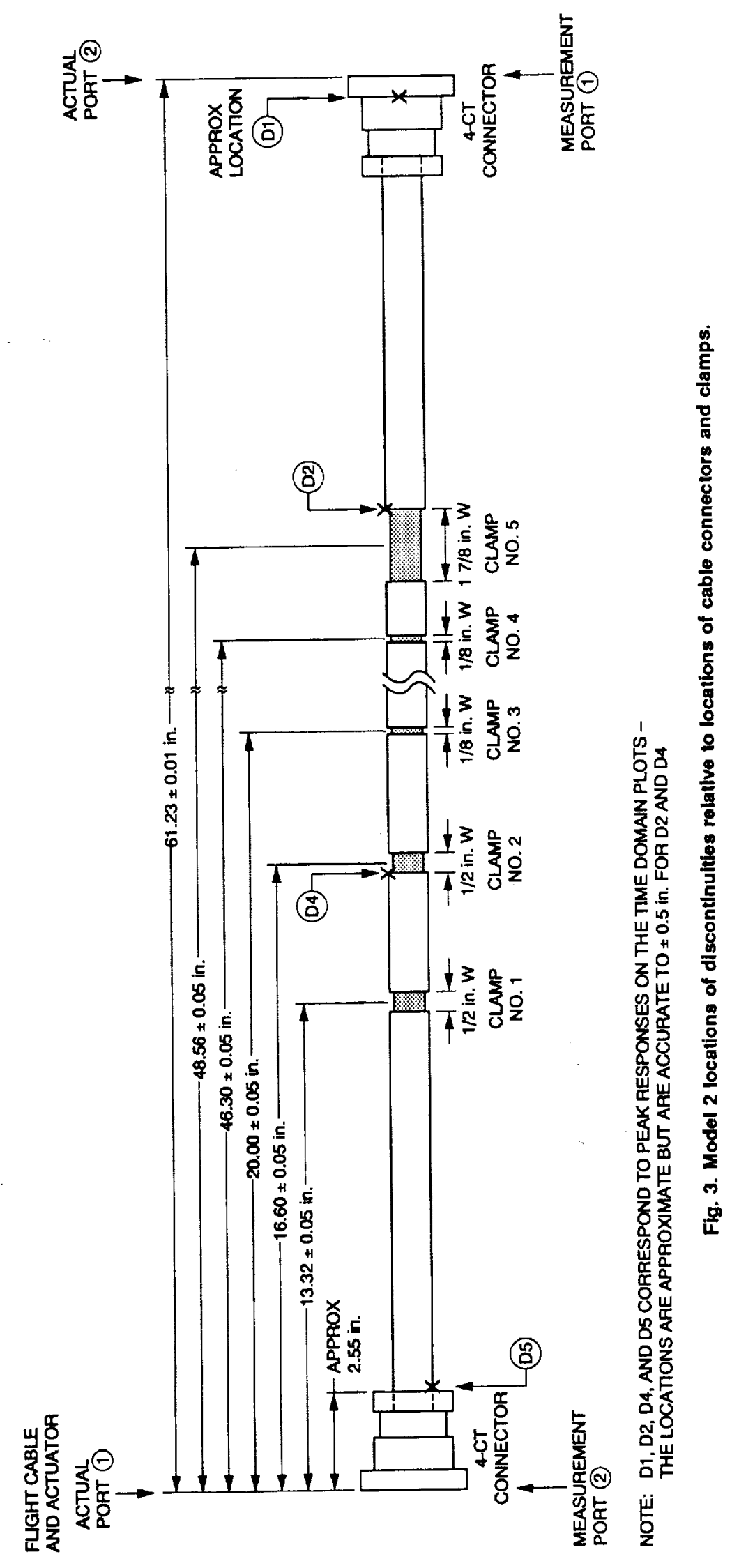




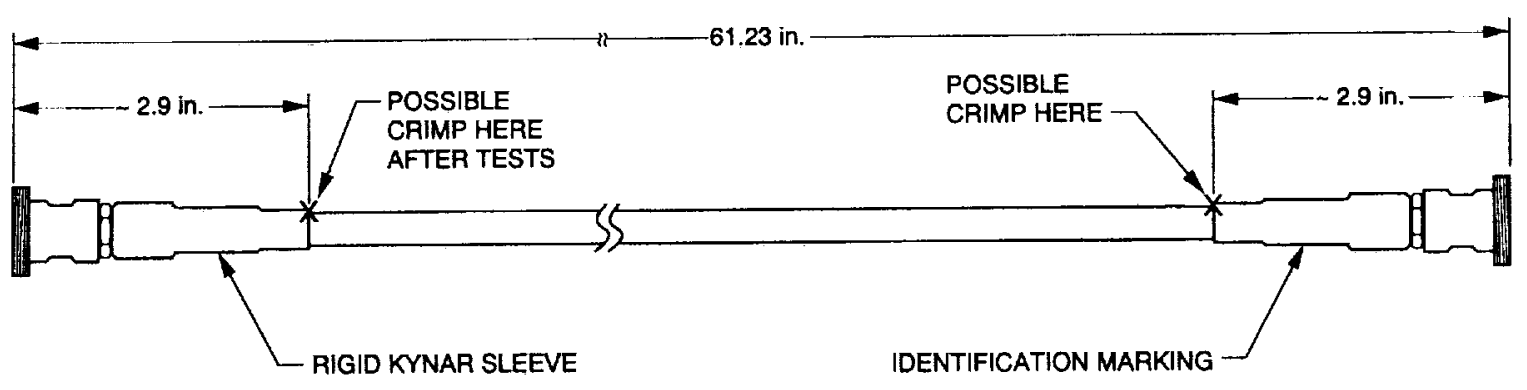

Fig. 4. Galileo S-band cable outer dimenslon detall.

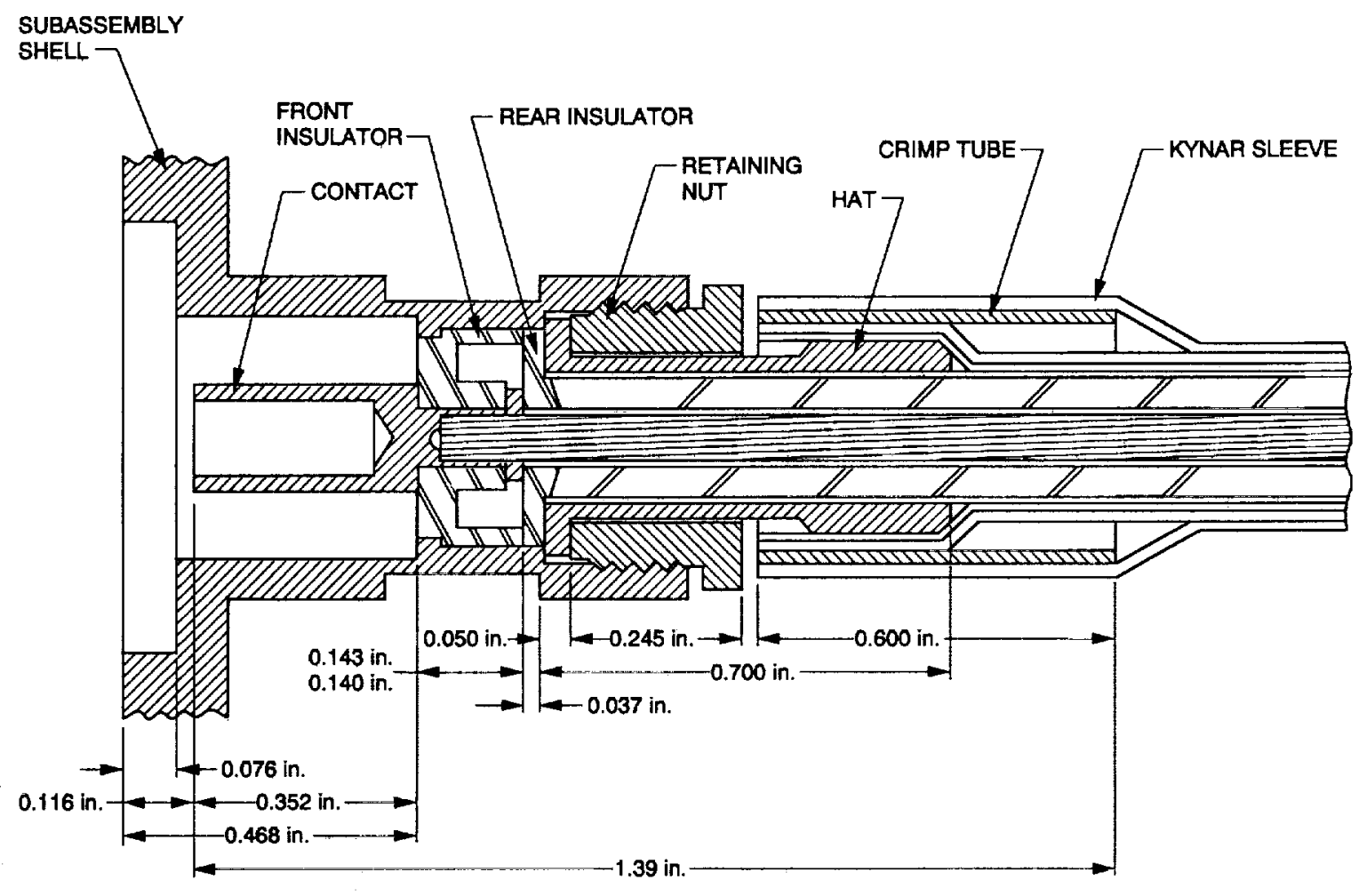

Flg. 5. Connector details. 


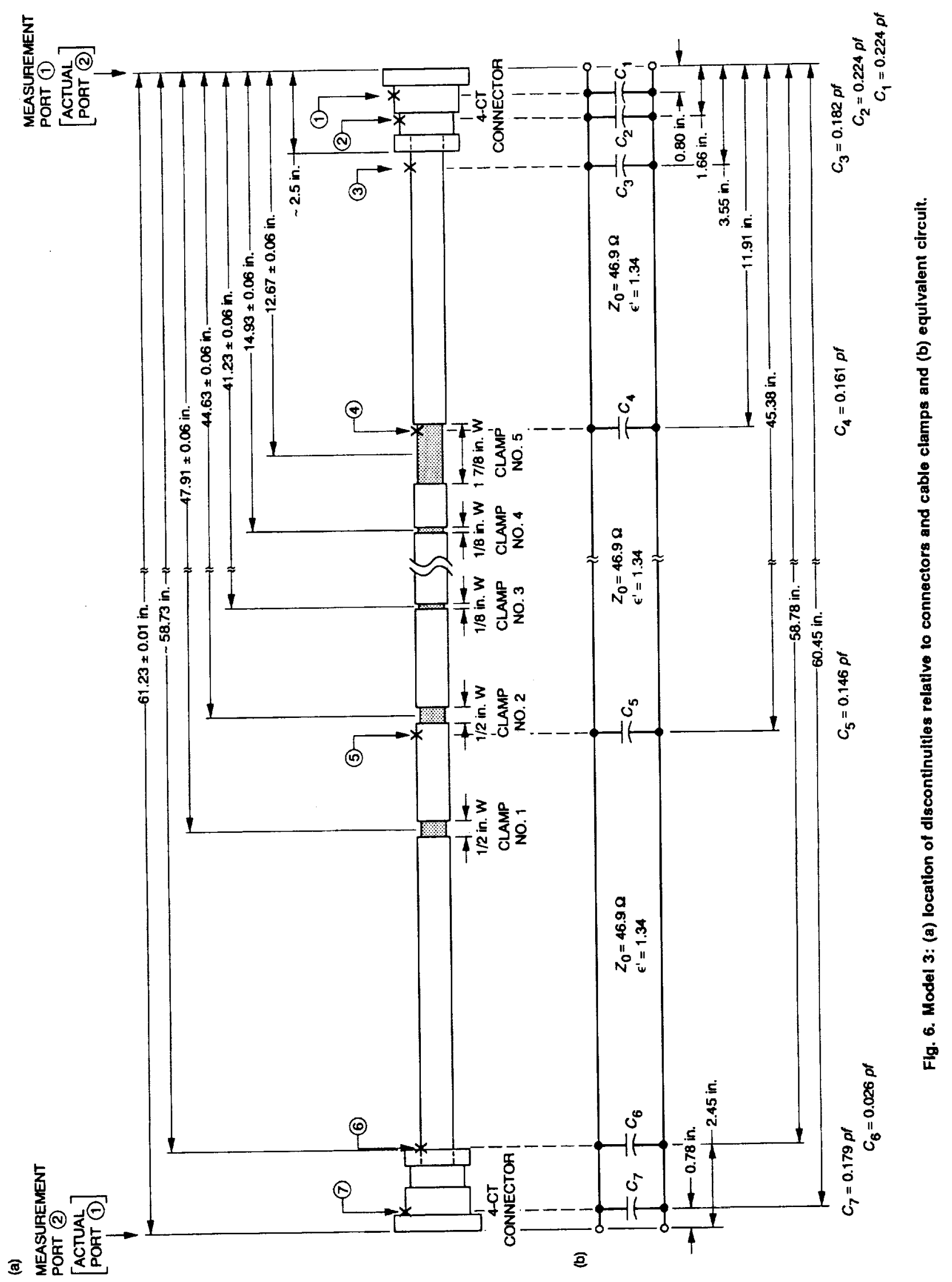




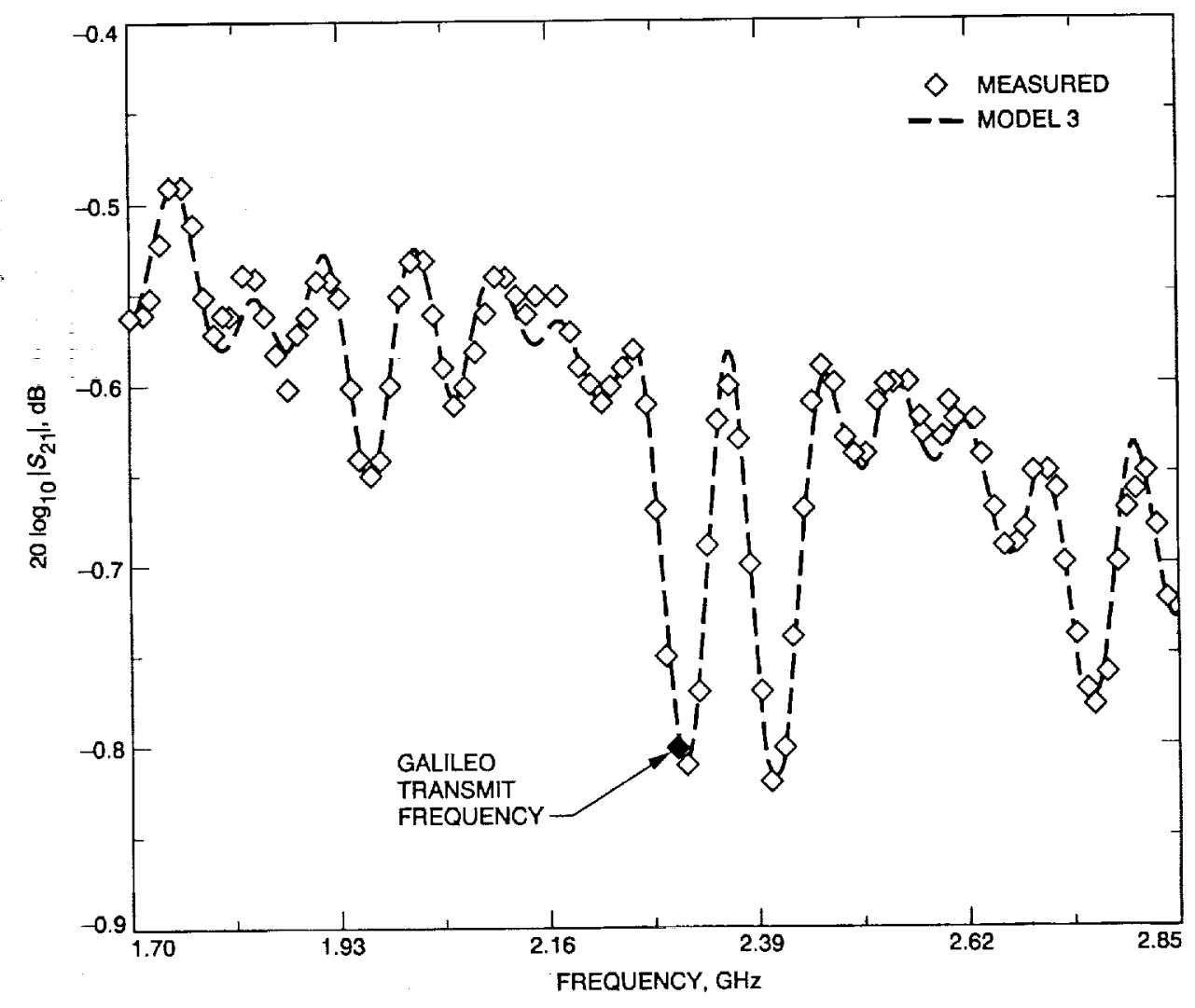

Fig. 7. Comparison of theoretical and measured Insertion losses for Model 3, assuming shunt capacliance values that are constant over the frequency range of Interest.

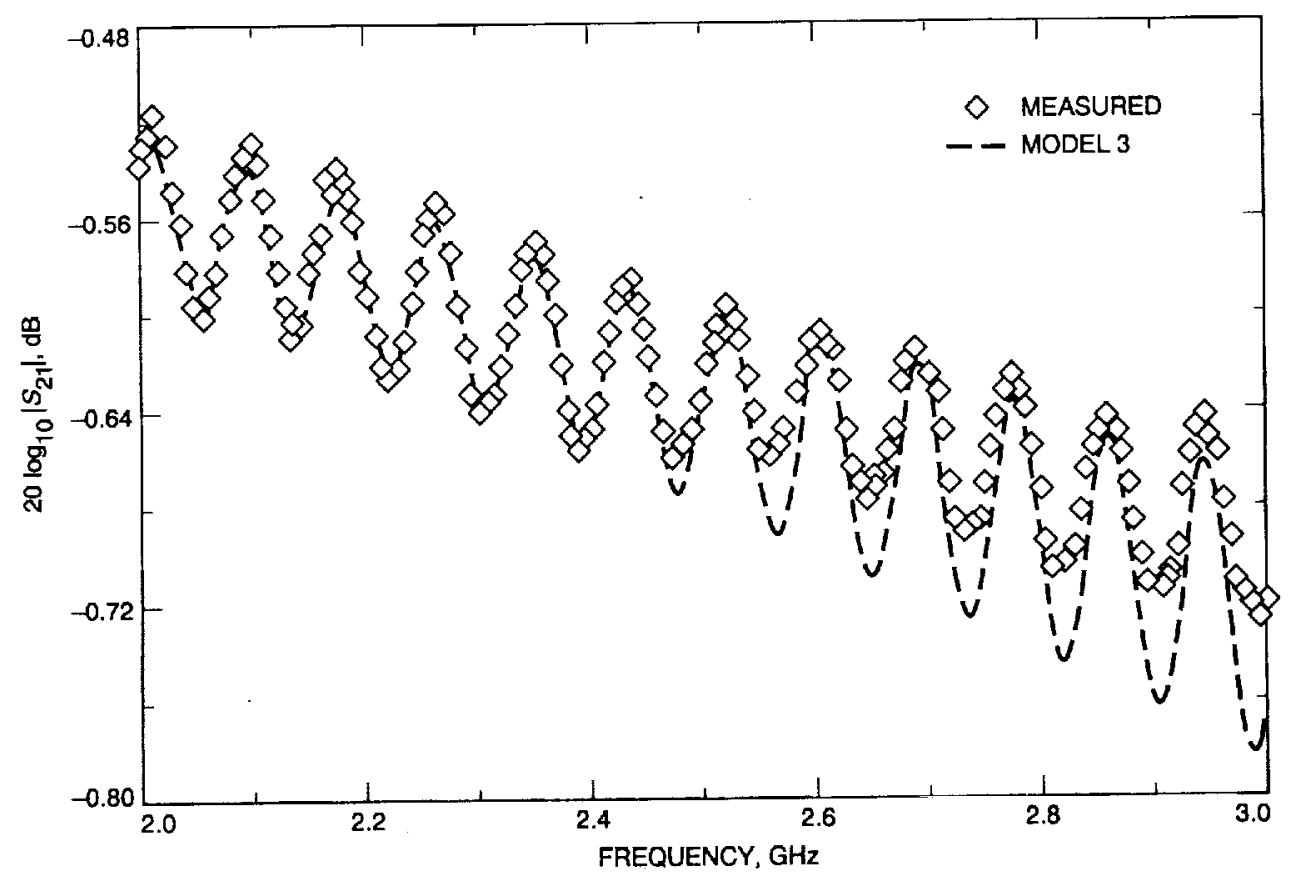

Fig. 8. Comparison of theoretical and measured insertion losses for Model 3 for the preenvironmental cable condition. 


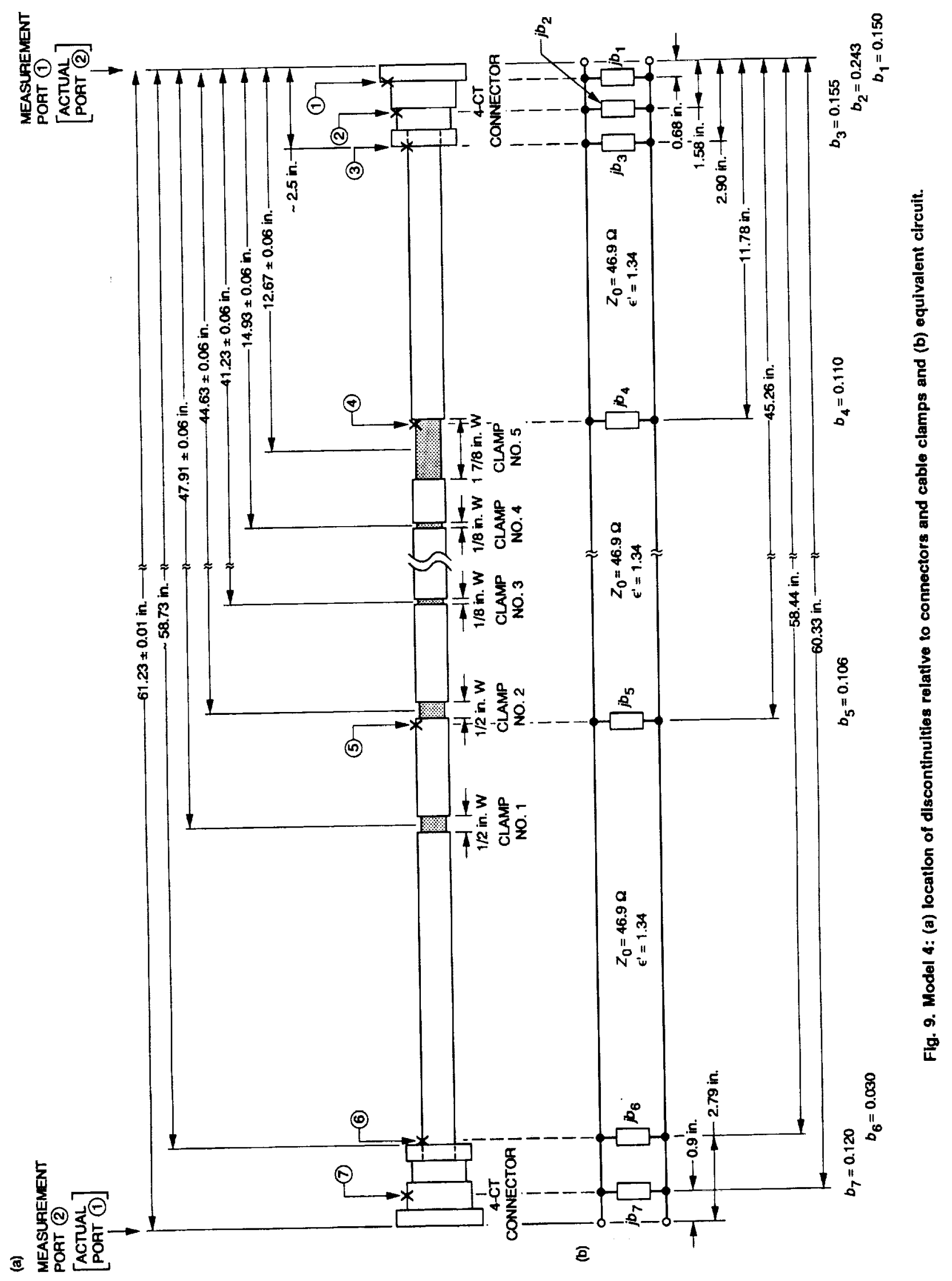




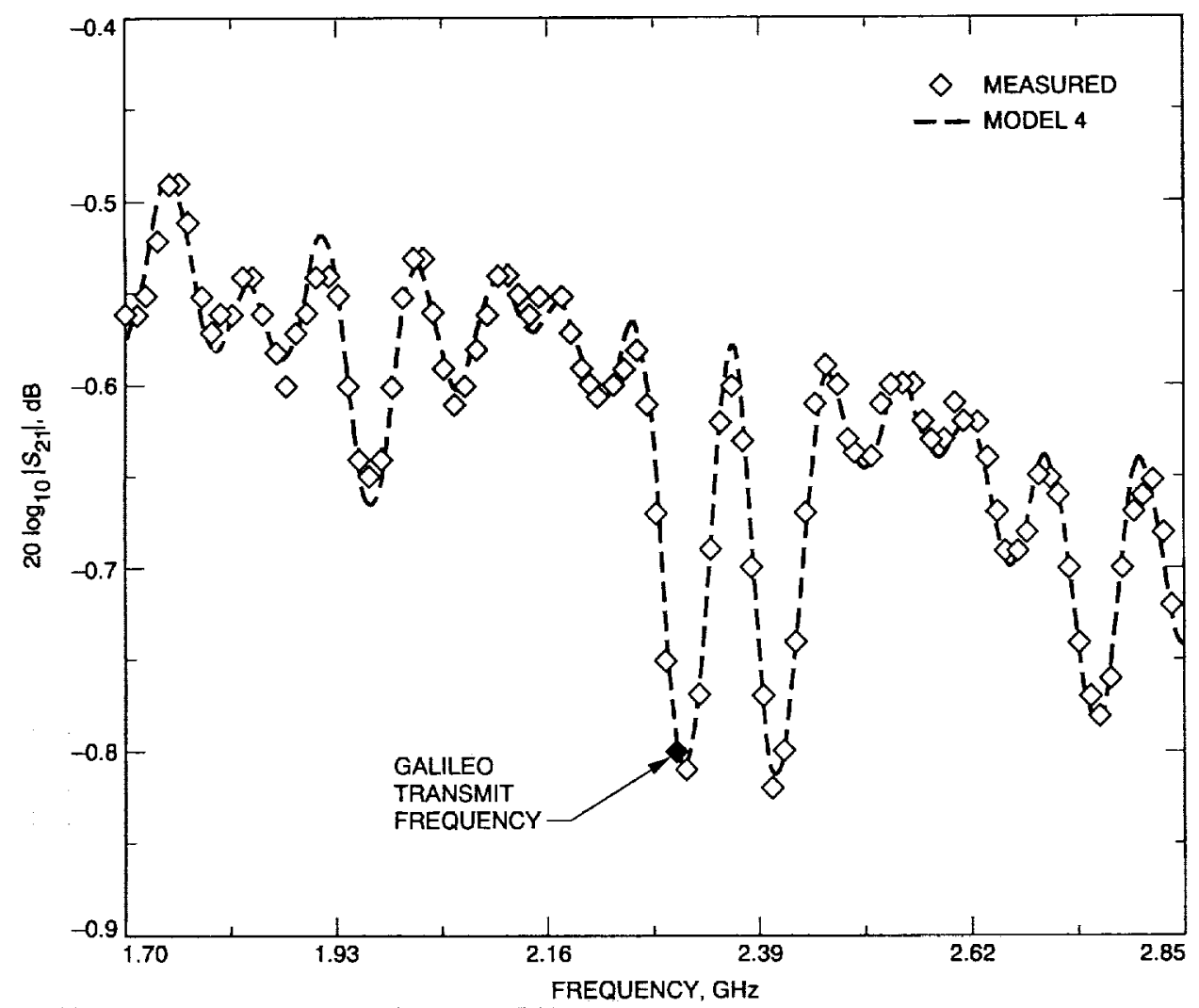

Fig. 10. Comparison of theoretical and measured Insertion losses for Model 4, assuming capacitlve shunt susceptance values that are constent over the frequency range of Interest.

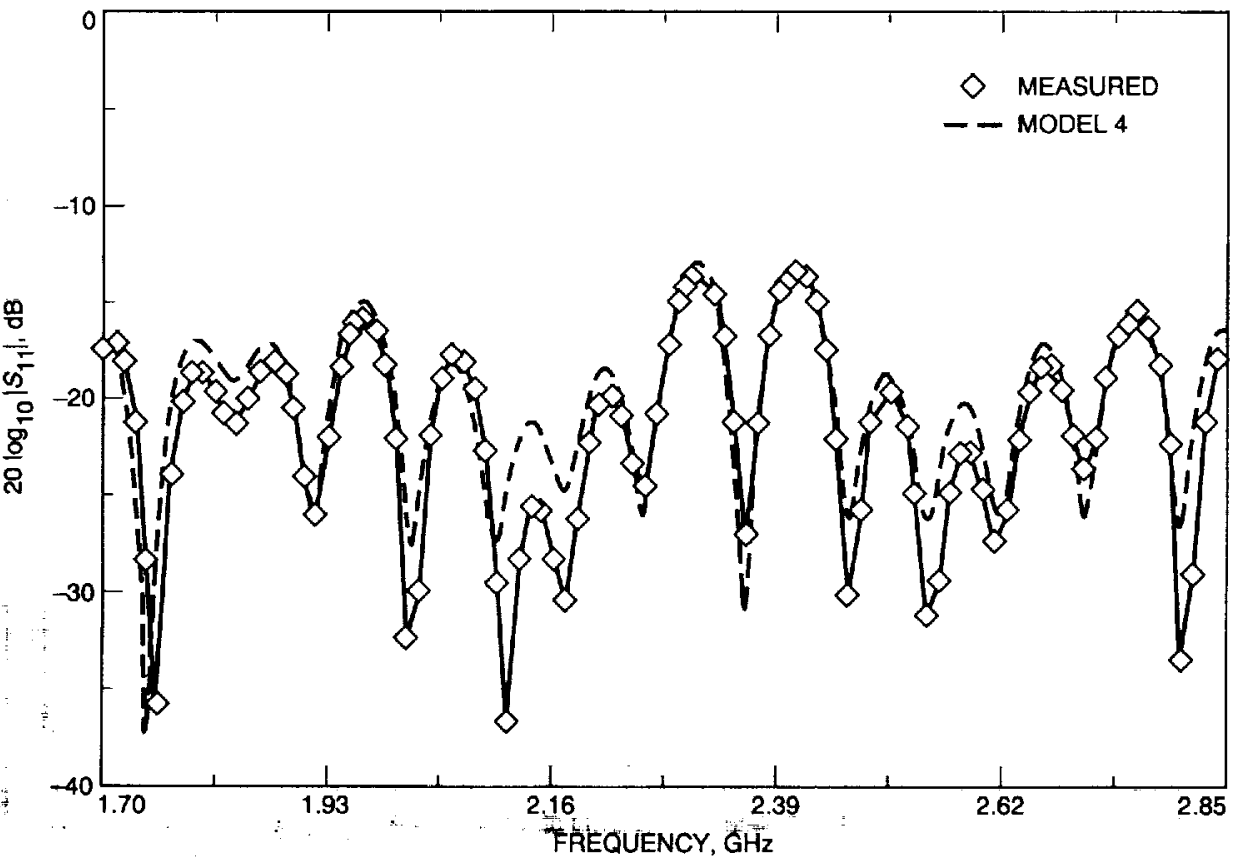

Fig. 11. Comparison of theoretical and measured return losses for Model 4, as seen looking into port 1. 


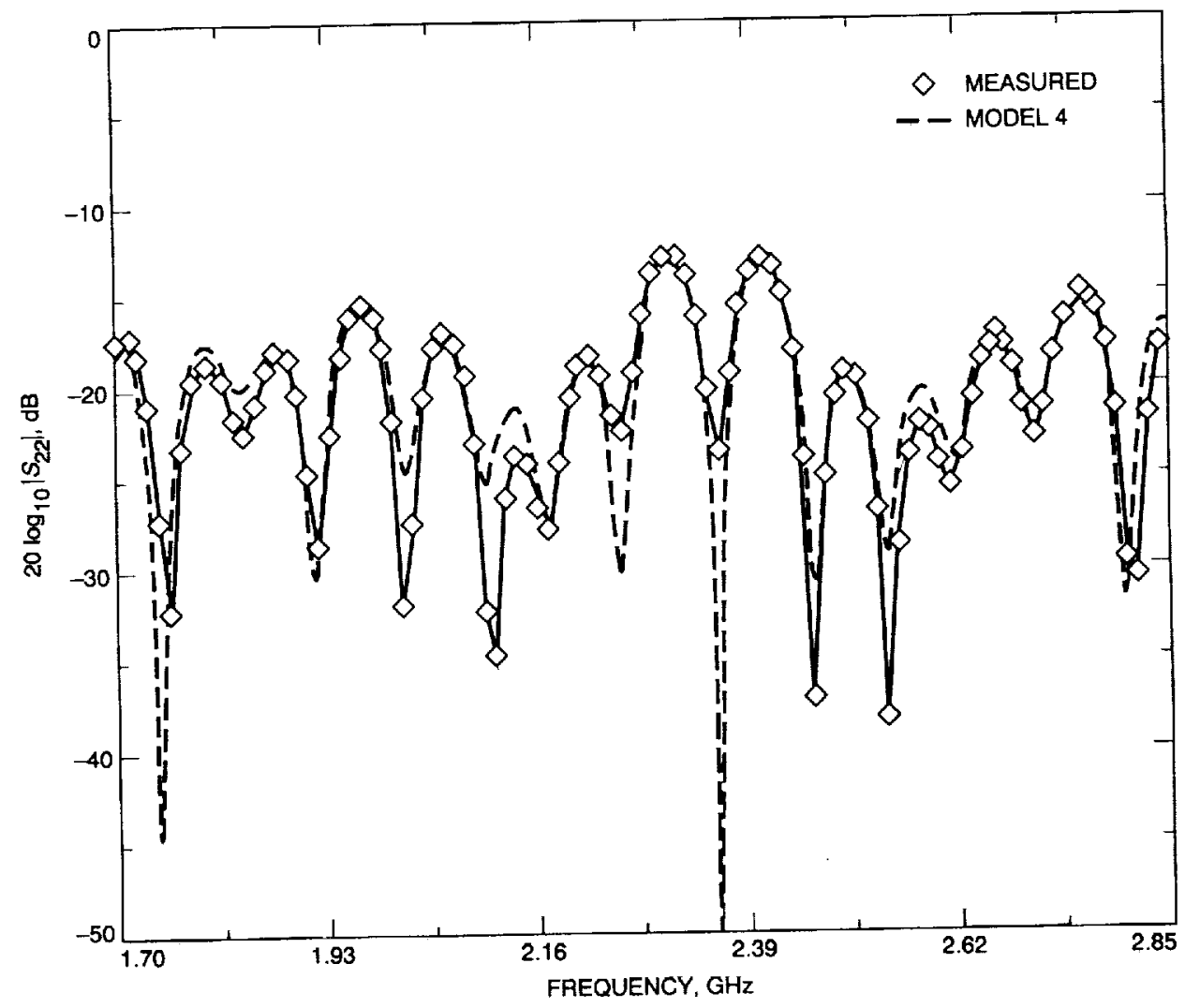

Flg. 12. Comparison of theoretical and messured return losses for Model 4, as seen looking Into port 2.

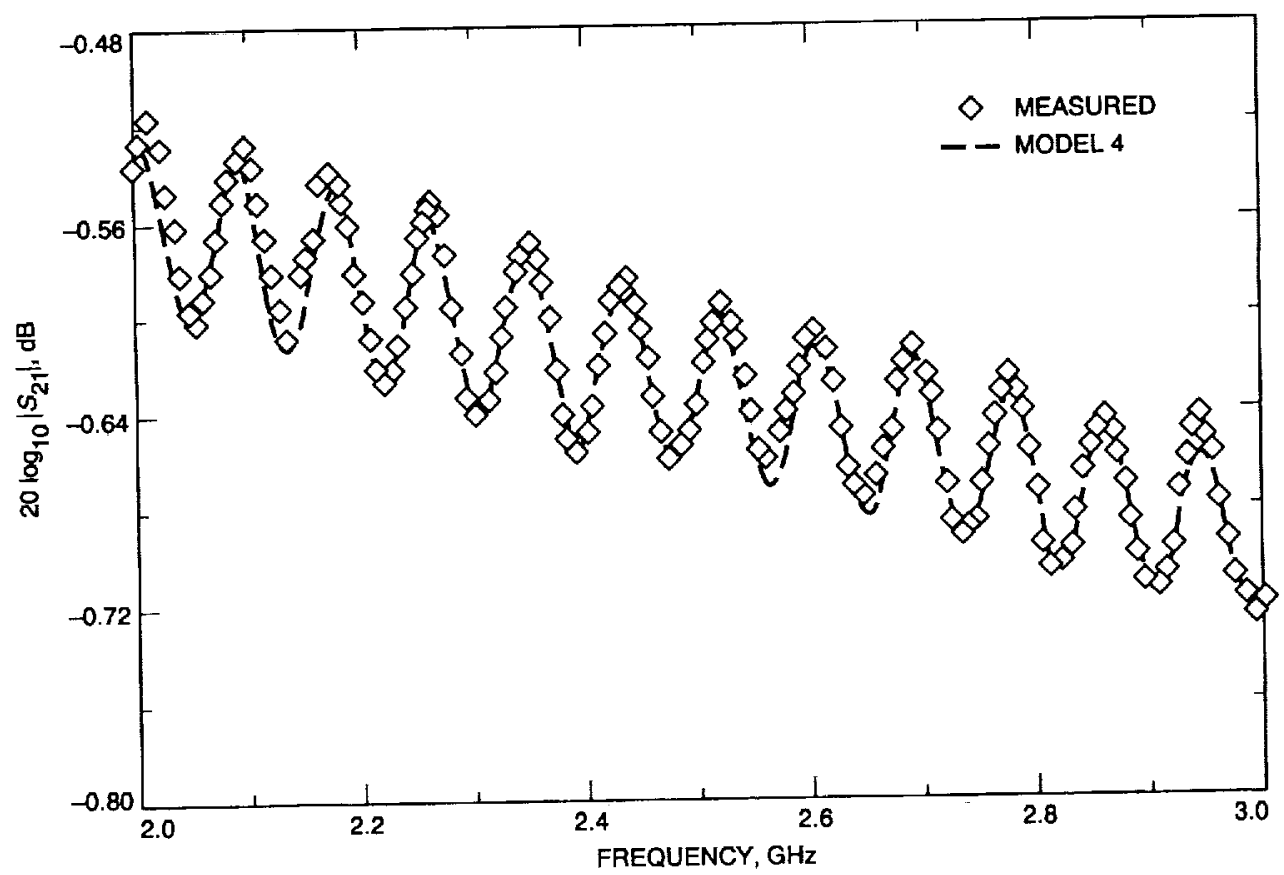

Fig. 13. Comparlson of theoretical and measured Insertion losses for Modal 4 for the preenvironmental cable condition. 


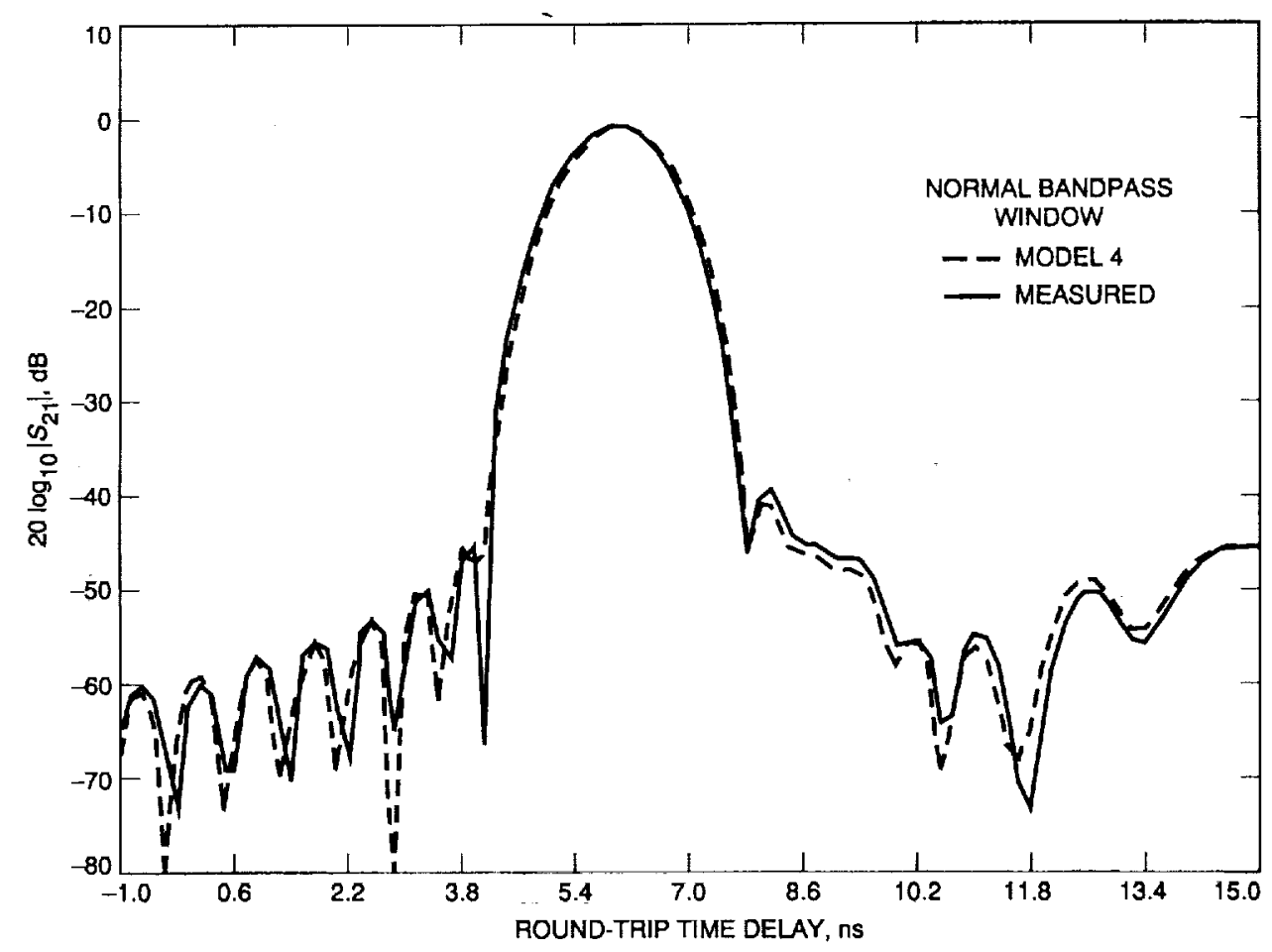

Fig. 14. Comparison of theoretical and measured time domain responses on $\left|s_{21}\right|$ data for Model 4.

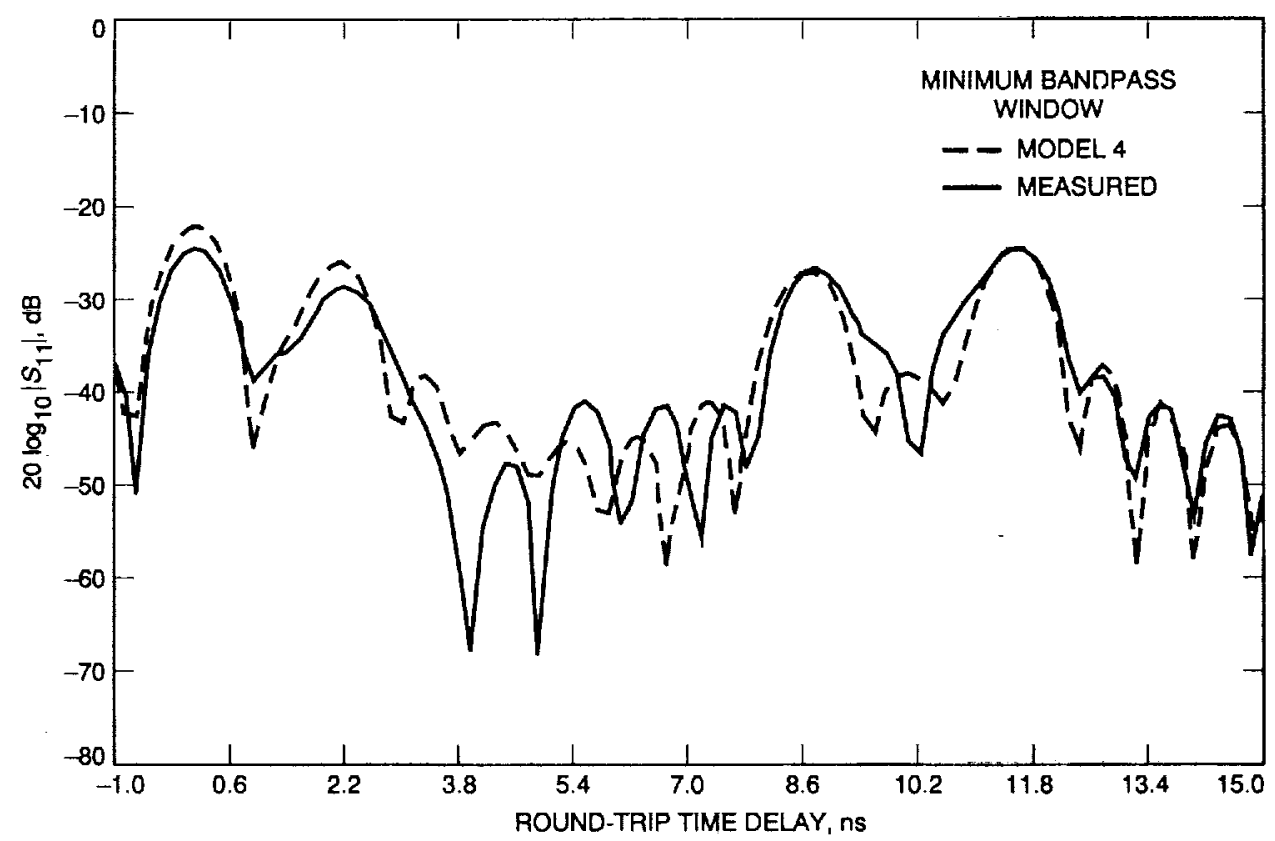

Flg. 15. Comparison of theoretical and measured time domain responses based on $\left|s_{11}\right|$ data for Model 4. The theoretical response curve had to be moved by the equivalent of 1.081 in. $(0.212$ nsec, round-trip) to the left in order to line up the peaks of curves. 


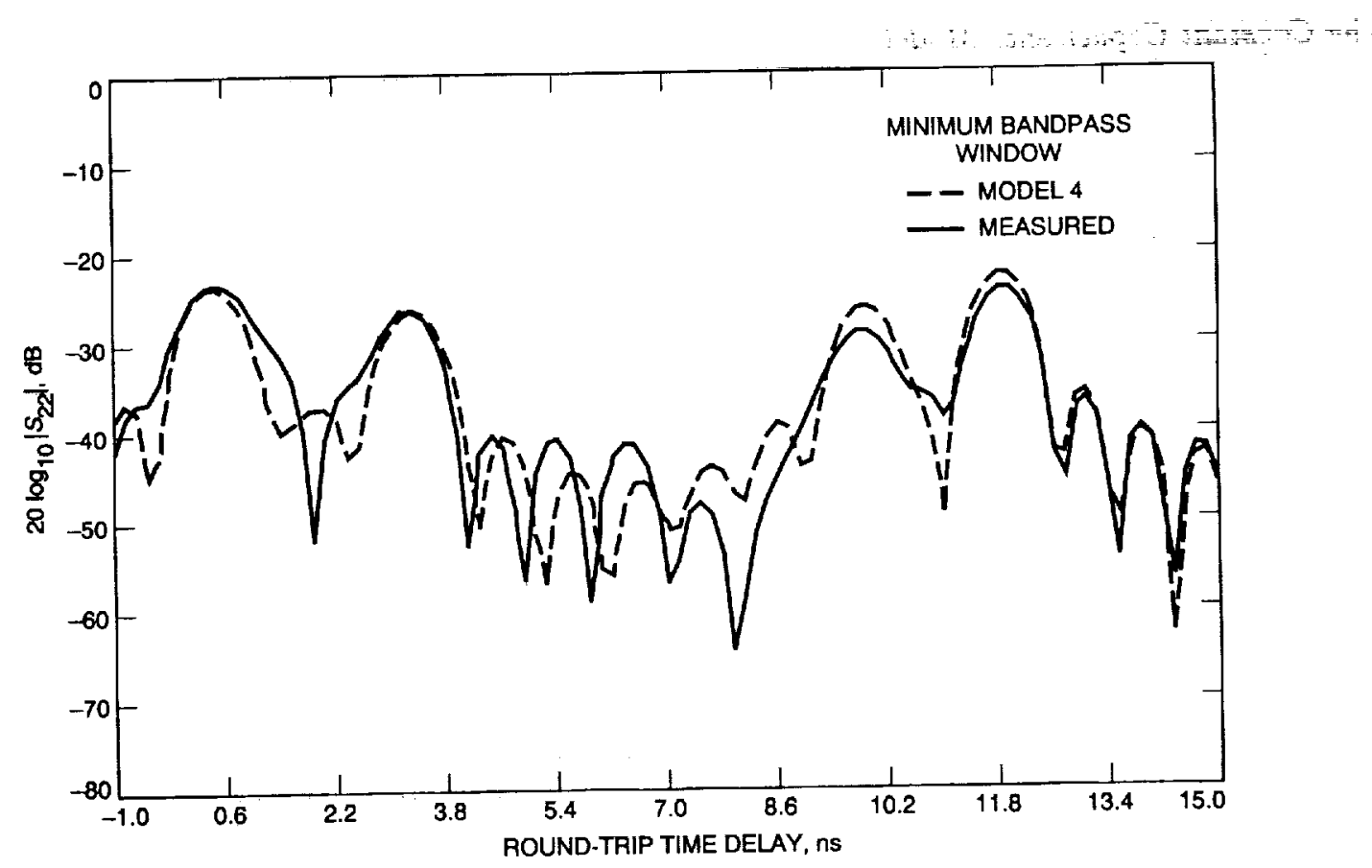

Flg. 16. Comparison of theoretical and measured time domain responses based on $\left|S_{22}\right|$ data for Model 4. The theoretical response curve had to be moved by the equivalent of 1.376 in. $(0.270$ nsec, round-trip) to the right in order to line up the peaks of curves. 


\section{Appendix A \\ S-Parameters of a Basic Network}

The basic network used in the modeling work is shown in Fig. A-1. The elements of the network are a capacitive shunt susceptance followed by a length of lossy transmission line. The $S$-parameters for this network are

$$
[S]=\frac{1}{2+j b}\left[\begin{array}{cc}
-j b & 2 e^{-\gamma \ell} \\
2 e^{-\gamma \ell} & -j b e^{-2 \gamma \ell}
\end{array}\right]
$$

where $b$ is the normalized shunt susceptance, $\gamma$ is the complex propagation constant and $\ell$ is the line length.

For the Constant Capacitance Model

$$
b=2 \pi f C Z_{0}
$$

where $f=$ frequency in hertz, $C$ is the capacitance in farads, and $Z_{0}$ is the transmission line characteristic impedance in ohms.

For the Constant Shunt Susceptance Model, $b=\mathrm{a}$ constant. The nominal value of $b$ for both models can be obtained from experimental return loss-time domain plots by using the relationship

$$
\begin{gathered}
b=\frac{2\left|S_{11}\right|}{\sqrt{1-\left|S_{11}\right|^{2}}} \\
\left|S_{11}\right|=10^{-\left(R L_{1} / 20-A_{\mathrm{dB}} / 10\right)}
\end{gathered}
$$

where $R L_{1}$ is the positive decibel return loss value measured at port 1 and $A_{\mathrm{dB}}$ is the positive decibel value for the transmission line loss between the discontinuity and port 1 . 

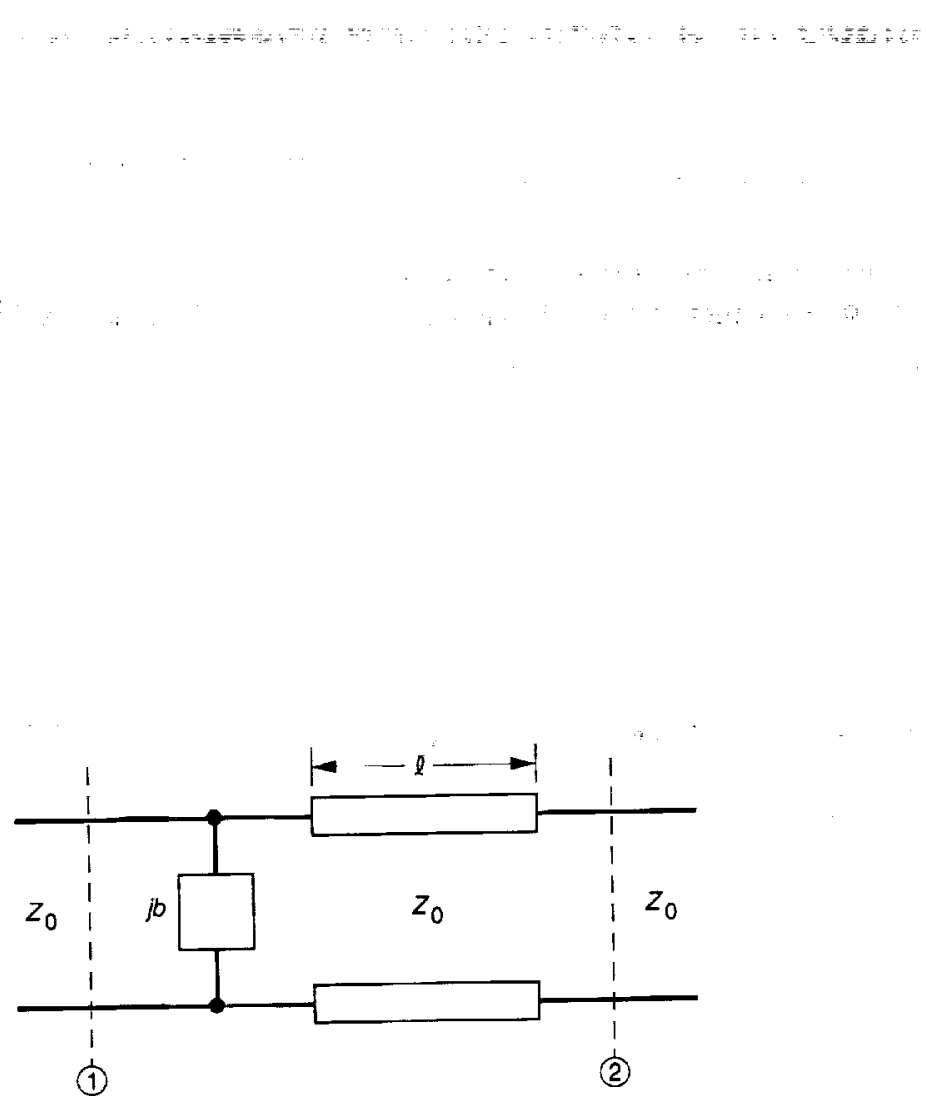

Fig. A-1. Basic network used in cable model. 


\section{Appendix B}

\section{A Method for Determining Cable-Only Attenuation From Measurements Made on a Cable Having Mismatched Connectors}

When a cable has connectors, the method normally used to determine the attenuation of a cable (minus its connectors) is to calculate it from the slope of the measured insertion loss versus frequency curve. When the connectors are mismatched, this method yields an insertion loss that is higher than the cable (minus connector) attenuation. Accurate determination of cable attenuation was critical for the modeling work that was done to obtain the results presented in this article.

From Table B-1, whose equations were derived from those given in [3],

$$
\begin{gathered}
\left|\ell_{11}\right| \approx \frac{\left|S_{11}\right|_{\max }+\left|S_{11}\right|_{\min }}{2} \\
\left|n_{22}\right| \approx \frac{\left|S_{22}\right|_{\max }+\left|S_{22}\right|_{\min }}{2} \\
\left|S_{21}\right|_{\max }=\frac{\sqrt{\left(1-\left|\ell_{11}\right|^{2}\right)\left(1-\left|n_{22}\right|^{2}\right)}}{1-h} e^{-\alpha \ell} \\
\left|S_{21}\right|_{\min }=\frac{\sqrt{\left(1-\left|\ell_{11}\right|^{2}\right)\left(1-\left|n_{22}\right|^{2}\right)}}{1+h} e^{-\alpha \ell}
\end{gathered}
$$

where

$$
h=\left|\ell_{11} n_{22}\right| e^{-2 \alpha \ell}
$$

Then from Eqs. (B-3) and (B-4), the average value of $\left|S_{22}\right|$ is derived as

$$
\begin{aligned}
\left|S_{21}\right|_{\text {avg }} & =\frac{\left|S_{21}\right|_{\max }+\left|S_{21}\right|_{\min }}{2} \\
& =\frac{\sqrt{\left(1-\left|\ell_{11}\right|^{2}\right)\left(1-\left|n_{22}\right|\right)^{2}}}{1-h^{2}} e^{-\alpha \ell}
\end{aligned}
$$

But $h^{2}$ is normally less than 0.01 and can be dropped from Eq. (B-5) so that

$$
\left|S_{21}\right|_{\text {avg }} \approx \sqrt{\left(1-\left|\ell_{11}\right|^{2}\right)\left(1-\left|n_{22}\right|^{2}\right)} e^{-\alpha \ell}
$$

then the cable (minus connector) attenuation is

$$
\begin{aligned}
A_{\mathrm{dB}}= & 20 \log _{10} e^{-\alpha \ell} \\
= & 20 \log _{10}\left|S_{21}\right|_{\text {avg }} \\
& -10 \log _{10}\left[\left(1-\left|\ell_{11}\right|^{2}\right)\left(1-\left|n_{22}\right|^{2}\right)\right]
\end{aligned}
$$

From the pre-environmental cable test data, it was found that near $2 \mathrm{GHz}$,

$$
\begin{aligned}
& \left|\ell_{11}\right| \approx 0.075 \\
& \left|n_{22}\right| \approx 0.066
\end{aligned}
$$

and the average loss of the cable shown in Fig. 13 between 2.015 and $2.055 \mathrm{GHz}$ is

$$
20 \log _{10}\left|S_{21}\right|_{\text {avg }}=-0.557 \mathrm{~dB}
$$

Substitution of these values into Eq. (B-7) gives $A_{\mathrm{dB}}$ equal to $-0.514 \mathrm{~dB}$. The contribution of the mismatched connectors from the last term on $\mathrm{Eq}$. (B-7) is about $0.043 \mathrm{~dB}$. For the Galileo cable, the length of the section without connectors is 59.647 in., so the attenuation of the cable (minus connectors) is $-0.00861 \mathrm{~dB} / \mathrm{in}$. This calculated value compares favorably with the stated manufacturer's data of approximately $-0.1 \mathrm{~dB} / \mathrm{ft}$ or $-0.00833 \mathrm{~dB} / \mathrm{in}$. However, it was not previously known that the manufacturer's approximate value was close to the true value. Although the corrections seem small, significantly better curve fits between experimental and theoretical data (for Models 3 and 4) were obtained when the more accurate values of the cable attenuation were used at each frequency. 

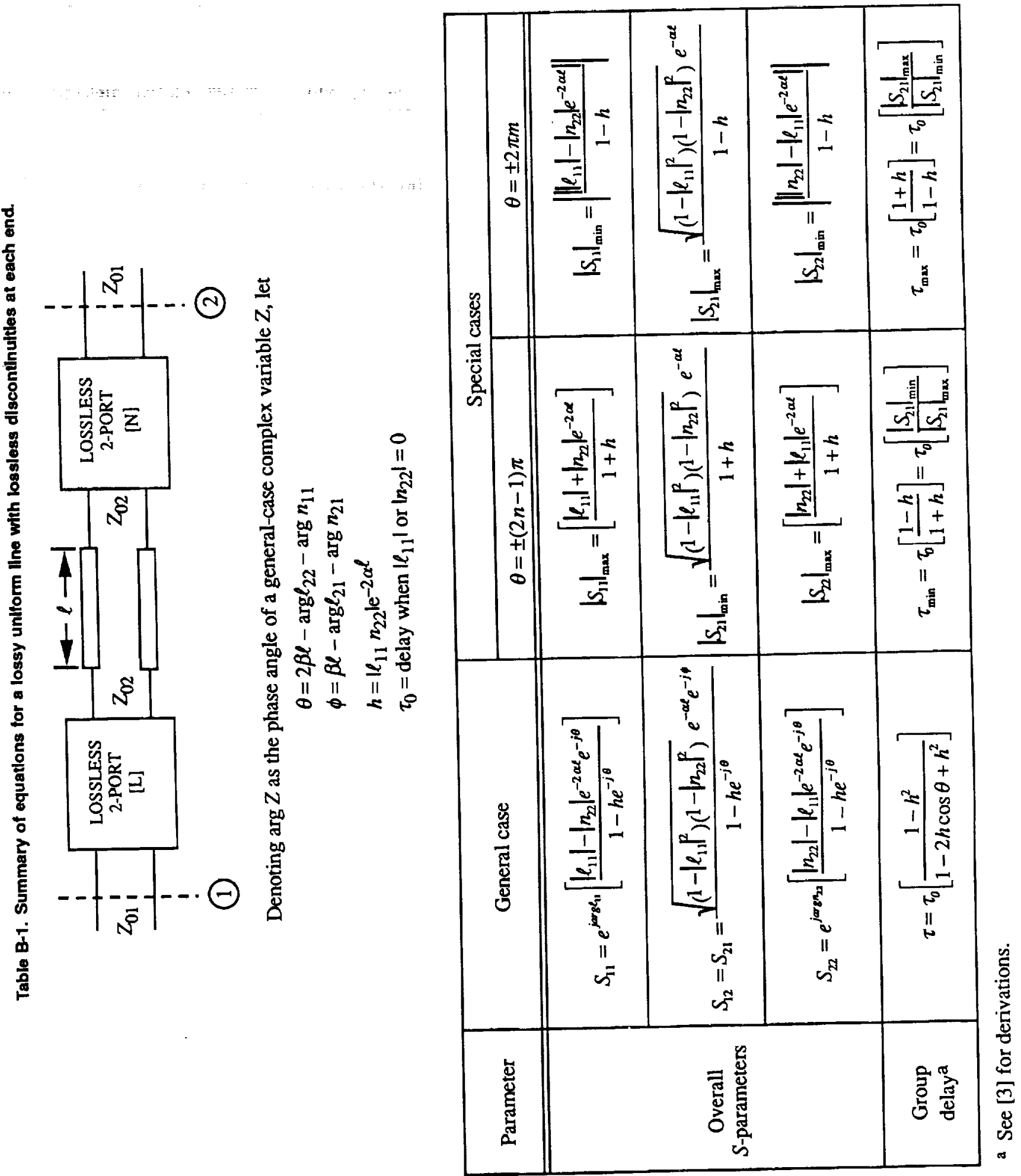


\section{Appendix C}

\section{Equivalent Circuits of a Cable With a Reduced Outer Diameter Section}

The equivalent circuits of the section of coaxial transmission line with a reduced outer diameter over length $\ell$ are shown in Fig. C-1. The characteristic impedance of the nominal and reduced sections $Z_{01}$ and $Z_{02}$, respectively, are

$$
\begin{aligned}
& Z_{01}=\frac{60}{\sqrt{\epsilon_{1}^{\prime}}} \ln \left(\frac{D_{\mathrm{O} 1}}{D_{\mathrm{I} 1}}\right) \\
& Z_{02}=\frac{60}{\sqrt{\epsilon_{2}^{\prime}}} \ln \left(\frac{\bar{D}_{\mathrm{O} 2}}{D_{12}}\right)
\end{aligned}
$$

where $D_{01}$ and $D_{02}$ are the diameters of the outer conductors for the nominal and reduced sections, respectively. The symbols $D_{11}$ and $D_{12}$ are the diameters of the inner conductors for the nominal and reduced sections, respectively, and $\epsilon_{1}^{\prime}$ and $\epsilon_{2}^{\prime}$ are the relative dielectric constants of the media in the nominal and reduced sections, respectively.

Using the equations for the equivalent circuit shown in Fig. C-1(b) given by Beatty [4], the value of $\left|S_{11}\right|$ can be calculated. That value is then used in Eq. (A-3) to compute an equivalent shunt susceptance corresponding to the equivalent circuit shown in Fig. C-2(c). If length $\ell$ is very short ( $<0.005$ wavelength), representing a crimp or deep crease in the outer cable, then the discontinuity should be represented as a shunt capacitive susceptance $b$ of constant value over the frequency range of interest. If the length $\ell$ is about ( $0.1-0.25$ wavelength), or about equal to the width of a cable clamp, then the equivalent $b$ should be represented as a shunt susceptance with a capacitance of constant value. 

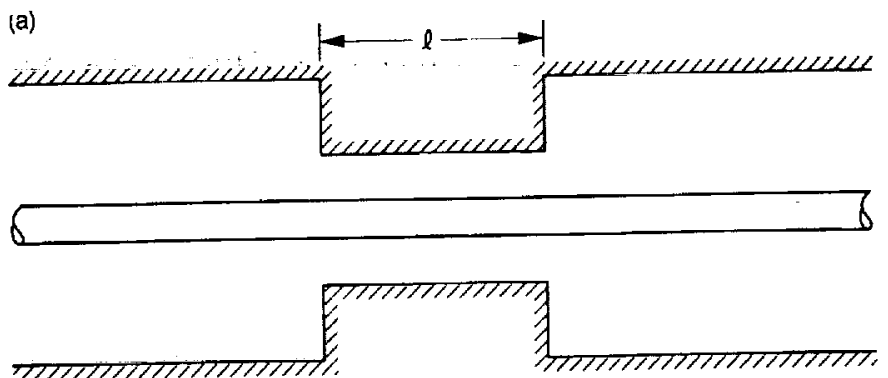

(b)

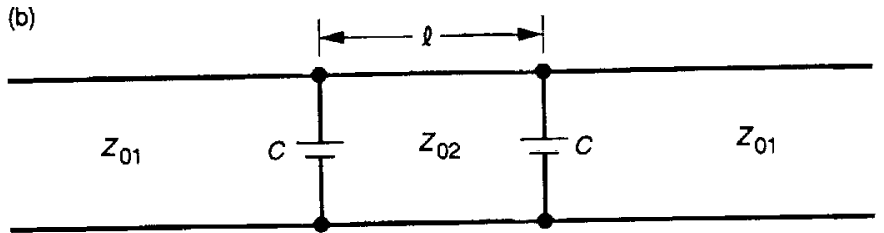

(c)

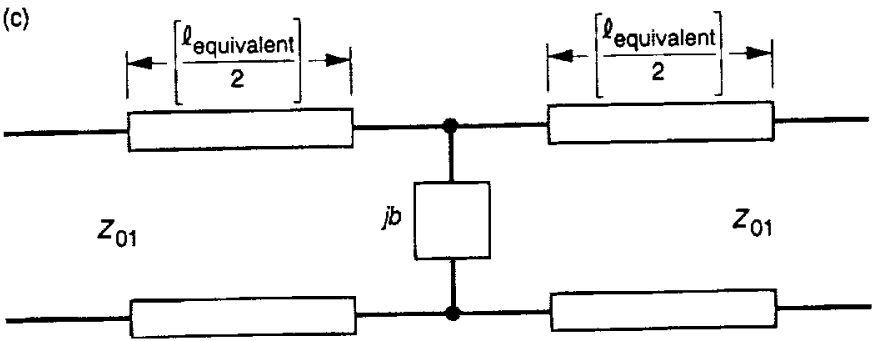

Fig. C-1. Cable with a section of reduced outer diameter: (a) physical representation; (b) equivalent circult with two capacitive shunt discontinuities separated by a line length of reduced section; and (c) equivalent circult with a single shunt discontinulty and equivalent line lengths. 


\section{Referénces}

[1] C. L. Lawson, "Nonlinear Least-Squares-Plain and Fancy," Computing and Information Services News, Jet Propulsion Laboratory, Pasadena, California, vol. 8, no. 4, pp. 6-7, April 1990.

[2] D. M. Kerns and R. W. Beatty, Basic Theory of Waveguides and Introductory Microwave Network Analysis, New York: Pergammon, pp. 42-43, 1967.

[3] R. W. Beatty and T. Y. Otoshi, "Effect of Discontinuities on the Group Delay of a Microwave Transmission Line," IEEE Trans. Microwave Theory and Techniques, vol. MTT-23, pp. 919-922, November 1975.

[4] R. W. Beatty, "Calculated and Measured $S_{11}$, and $S_{21}$, and Group Delay for Simple Types of Coaxial and Rectangular Waveguide 2-Port Standards," NBS Technical Note 657, National Bureau of Standards, Boulder, Colorado, p. 18, December 1974. 